\title{
SIMPLEST GRAPHS WITH SMALL EDGES: ASYMPTOTICS FOR RESOLVENTS AND HOLOMORPHIC DEPENDENCE OF SPECTRUM
}

\author{
D.I. BORISOV, M.N. KONYRKULZHAEVA
}

\begin{abstract}
In the work we consider a simplest graph formed by two finite edges and a small edge coupled at a common vertex. The length of the small edge serves as a small parameter. On such graph, we consider the Schrödinger operator with the Kirchoff condition at the internal vertex, the Dirichlet condition on the boundary vertices of finite edges and the Dirichlet or Neumann condition on the boundary vertex of the small edge. We show that such operator converges to a Schrödinger operator on the graph without the small edge in the norm resolvent sense; at the internal vertex one has to impose the Dirichlet condition if the same was on the boundary vertex of the small edge. If the boundary vertex was subject to the Neumann condition, the internal vertex keeps the Kirchoff condition but the coupling constant can change. The main obtained result for the resolvents is the two-terms asymptotics for their resolvents and an estimate for the error term.

The second part of the work is devoted to studying the dependence of the eigenvalues on the small parameter. Despite the graph is perturbed singularly, the eigenvalues are holomorphic in the small parameter and are represented by convergent series. We also find out that under the perturbation, there can be stable eigenvalues independent of the parameter. We provide a criterion determining the existence of such eigenvalues. For varying eigenvalues we find the leading terms of their Taylor series.
\end{abstract}

Keywords: Graph, small edge, spectrum, asymptotics

Mathematics Subject Classification: 34B45, 81Q15

\section{INTRODUCTION}

During the last twenty years, the spectra of elliptic operators on graphs, or simply the theory of quantum graphs, is being intensively developed and there is a huge number of works on this subject. Not trying to mention all works, we just cite the monographs [5], [7] and the references therein.

An important direction of studies is the developing of the perturbation theory for quantum graphs. And one of the most interesting perturbations due to the specific geometry of graphs is small edges. The study of such problems was initiated rather recently. It was shown in work [9] that an arbitrary boundary condition at a vertex can be approximated in the norm resolvent sense by means of a graph with small edges and a $\delta$-interaction in internal vertices. The most detailed study of the graphs with small edges was made in a recent work [8]. Here graphs of arbitrary structure with arbitrary boundary conditions at the vertices were considered and some of the edges were assumed to be of a small length. The norm resolvent convergence and

D.I. Borisov, M.N. Konyrkulzhaeva, Simplest graphs With SMAll EdGes: ASYmptotics For RESOLVENTS AND HOLOMORPHIC DEPENDENCE OF SPECTRUM.

(c) D.I. Borisov, Konyrkulzhaeva M.N. 2018.

The reported study by D.I. Borisov was funded by Russian Foundation for Basic Research according project no. 18-01-00046.

Submitted January 5, 2019. 
the convergence of the spectra were studied in great details depending on the structure of the graphs and of given boundary conditions.

The results of work [8] give rise to the following natural issues: how the asymptotics of the resolvents of operators on graphs with small edges looks like? What is the dependence of the spectrum on the lengths of small edges, for instance, how do the eigenvalues depend on them? Of course, the answers for these questions are to be found first for simple graphs and a simplest model seems to be a star-shaped graph with three edges, one of which is of a small length, see Figure 1. Surprisingly, but even such simple model features rather unexpectable properties. In our opinion, these properties deserve a separate study and exactly this is done in the present paper.

The main obtained results are as follows. In the internal vertex of the considered graph we impose a $\delta$-interaction, the boundary vertices of finite edges are subject to the Dirichlet condition, while on the boundary edge of the small the Neumann condition is imposed. The length of the small edge serves as a small parameter. As an operator, we choose a Schrödinger operator and at the small edge, a singular dependence of the potential on the small parameter is admitted. The limiting operator is the same Schrödinger operator but on a graph without the small edge, see Figure 2. In the case of the Dirichlet condition on the boundary vertex of the small edge, in the limit, the $\delta$-interaction in the internal vertex is replaced by the Dirichlet condition. In the case of the Neumann condition on the boundary vertex of the small edge, the $\delta$-interaction in the internal vertex is kept but in the coefficient there arises an additional term equalling to the mean value of the singular potential on the small edge. In both cases we obtain the leading terms in the asymptotics of the resolvents and we estimate an error term in the sense of the operator norm of the resolvent. At that, it turns out that the error is estimated only by $L_{2}$-norm on the small edge of a function, at which the resolvents of the original and limiting operators act, see Theorems 2.1, 2.2.

We also study the behavior of the eigenvalue with respect to the small parameter. We find out that the eigenvalues of the considered graphs with small edge are holomorphic in the small parameter. This is a rather unexpected result in view of the fact that a small edge is a singular perturbation and as a rule, under such perturbations, one can write out asymptotic series for the eigenvalues but usually, these series diverge. Moreover, our model turns out to have stable eigenvalues independent of the small parameter. For moving eigenvalues we find explicitly the leading terms of their Taylor series and in the case of the Dirichlet condition on the boundary vertex of the small edge the first correctors turn out to be negative.

The paper is organized as follows. In the next section we introduce main notations and formulate the main results. In the third and fourth sections we construct the leading terms in the asymptotics for the resolvents. The behavior of the eigenvalues is studied in the fifth and the sixth sections.

\section{Formulation OF PROBLEM AND MAIN RESUlts}

Let $\Gamma_{\varepsilon}$ be an oriented graph formed by three finite edges, one internal vertex connecting these edges and three boundary edges. Two edges are chosen to be of fixed lengths, while the third edge is assumed to be small, see Figure 1. We denote the edges of the graph by $e_{-}, e_{+}$, $e_{\varepsilon}$ with lengths $a_{-}, a_{+}$and $\varepsilon$, respectively, where $\varepsilon$ is a small positive parameter. On the edges we introduce respectively variables $x_{ \pm} \in\left[0, a_{ \pm}\right]$and $x_{\varepsilon} \in[0, \varepsilon]$. The internal vertex is denoted by $M_{0}$; we suppose that it corresponds to $x_{ \pm}=0, x_{\varepsilon}=0$. The boundary vertices correspond to the values $x_{ \pm}=a_{ \pm}, x_{\varepsilon}=\varepsilon$ and are denoted by $M_{ \pm}$and $M_{\varepsilon}$.

In the space $L_{2}\left(\Gamma_{\varepsilon}\right):=L_{2}\left(e_{-}\right) \oplus L_{2}\left(e_{+}\right) \oplus L_{2}\left(e_{\varepsilon}\right)$ we introduce the Schrödinger operator

$$
-\frac{d^{2}}{d x^{2}}+V_{\varepsilon}(x)+\alpha \delta(x)
$$




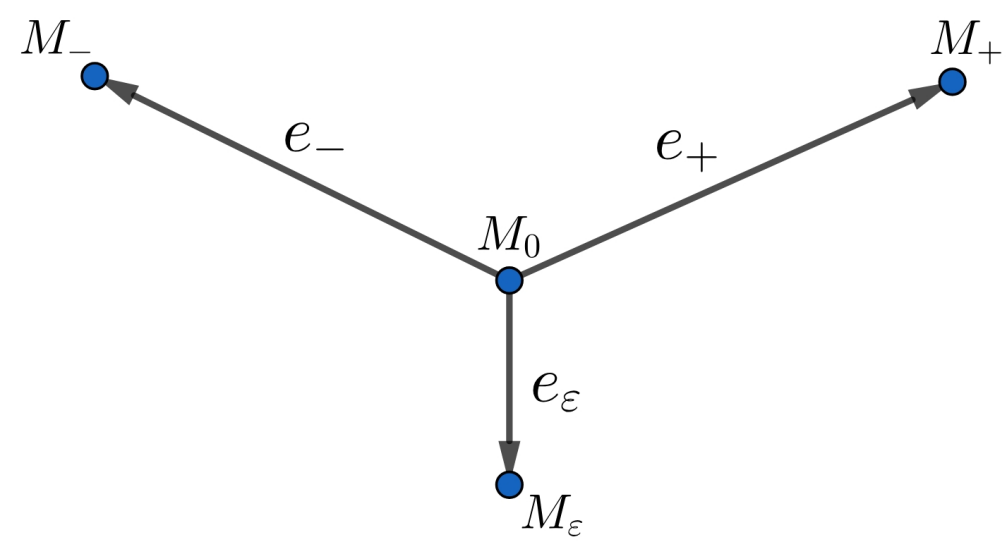

FIGURE 1. Graph $\Gamma_{\varepsilon}$ with a small edge: the lengths of $e_{ \pm}$are equal to fixed numbers $a_{ \pm}$, the length of $e_{\varepsilon}$ is equal to $\varepsilon$ being a small parameter

where the derivatives are taken with respect to the variables on the edges and the potential $V_{\varepsilon}$ is defined by the identity

$$
V_{\varepsilon}(x):=\left\{\begin{array}{cc}
W_{-}\left(x_{-}\right) & \text {on } e_{-}, \\
W_{+}\left(x_{+}\right) & \text {on } e_{+}, \\
\varepsilon^{-1} W_{-1}\left(\frac{x_{\varepsilon}}{\varepsilon}\right)+W_{0}\left(\frac{x_{\varepsilon}}{\varepsilon}\right) & \text { on } e_{\varepsilon} .
\end{array}\right.
$$

Here $W_{ \pm}$are real bounded measurable functions on $e_{ \pm}, W_{-1}, W_{0}$ are real bounded measurable functions on $[0,1]$. The last term in (2.1) describes a $\delta$-interaction with the coupling constant $\alpha \in \mathbb{R}$ and it corresponds to the following boundary condition in the internal vertex:

$$
u_{-}(0)=u_{+}(0)=u_{\varepsilon}(0)=: u\left(M_{0}\right), \quad u_{-}^{\prime}(0)+u_{+}^{\prime}(0)+u_{\varepsilon}^{\prime}(0)=\alpha u\left(M_{0}\right),
$$

where $u=\left(u_{-}, u_{+}, u_{\varepsilon}\right)$ is a function defined on the graph $\Gamma_{\varepsilon}$. On the boundary vertices $M_{ \pm}$we impose the Dirichlet condition

$$
u_{ \pm}\left(M_{ \pm}\right)=0
$$

while the vertex $M_{\varepsilon}$ is subject to the Dirichlet condition

$$
u_{\varepsilon}(\varepsilon)=0
$$

or to the Neumann condition

$$
u_{\varepsilon}^{\prime}(\varepsilon)=0 .
$$

We denote the introduced operator by $\mathcal{H}_{\varepsilon}^{D}$ in the case of boundary condition 2.4 and by $\mathcal{H}_{\varepsilon}^{R}$ in the case of boundary condition (2.5). As the domains of the operators $\mathcal{H}_{\varepsilon}^{D}$ and $\mathcal{H}_{\varepsilon}^{R}$, we choose the following dense in $L_{2}\left(\Gamma_{\varepsilon}\right)$ subsets:

$$
\begin{aligned}
& \mathfrak{D}\left(\mathcal{H}_{\varepsilon}^{D}\right):=\left\{u=\left(u_{-}, u_{+}, u_{\varepsilon}\right) \in L_{2}\left(\Gamma_{\varepsilon}\right): u_{ \pm} \in W_{2}^{2}\left(e_{ \pm}\right), u_{\varepsilon} \in W_{2}^{2}\left(e_{\varepsilon}\right),\right. \\
& \text { conditions 2.2), 2.3), 2.4 hold }\} \text {, } \\
& \mathfrak{D}\left(\mathcal{H}_{\varepsilon}^{R}\right):=\left\{u=\left(u_{-}, u_{+}, u_{\varepsilon}\right) \in L_{2}\left(\Gamma_{\varepsilon}\right): u_{ \pm} \in W_{2}^{2}\left(e_{ \pm}\right), u_{\varepsilon} \in W_{2}^{2}\left(e_{\varepsilon}\right)\right. \text {, } \\
& \text { conditions 2.2, 2.3, 2.5 hold }\} \text {. }
\end{aligned}
$$

The operators $\mathcal{H}_{\varepsilon}^{D}$ and $\mathcal{H}_{\varepsilon}^{R}$ are self-adjoint.

The main aim of our work is to study the behavior of resolvents and spectra of the operators $\mathcal{H}_{\varepsilon}^{D}$ and $\mathcal{H}_{\varepsilon}^{R}$ for small $\varepsilon$.

To formulate the main results, we shall need auxiliary notations. By $\Gamma_{0}$ we denote the graph obtained from $\Gamma_{\varepsilon}$ by removing the edge $e_{\varepsilon}$ and vertex $M_{\varepsilon}$, that is, the graph $\Gamma_{0}$ consists of two edges $e_{-}$and $e_{+}$coupled by the vertex $M_{0}$ and two boundary vertices $M_{ \pm}$, see Figure 2 . 


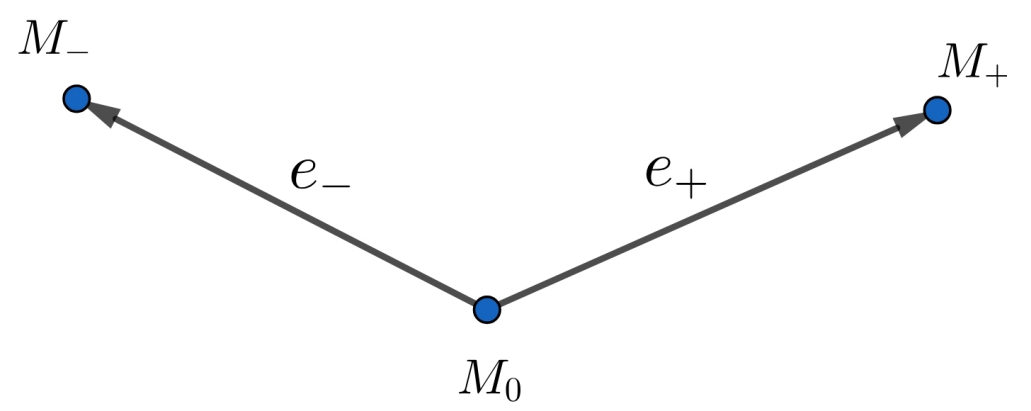

FIGURE 2. Limiting graph $\Gamma_{0}$

In the space $L_{2}\left(\Gamma_{0}\right):=L_{2}\left(e_{-}\right) \oplus L_{2}\left(e_{+}\right)$we consider the operator with the differential expression

$$
-\frac{d^{2}}{d x^{2}}+V_{0}, \quad V_{0}:=W_{ \pm} \quad \text { on } \quad e_{ \pm}
$$

subject to the Dirichlet condition at the boundary vertices $M_{ \pm}$:

$$
u_{ \pm}\left(M_{ \pm}\right)=0 .
$$

At the vertex $M_{0}$ we impose either the Dirichlet condition

$$
u_{ \pm}(0)=0
$$

or a delta-interaction:

$$
u_{-}(0)=u_{+}(0)=: u(0), \quad u_{+}^{\prime}(0)+u_{-}^{\prime}(0)=(\alpha+\beta) u(0), \quad \beta:=\int_{0}^{1} W_{-1}(t) d t .
$$

In the case of condition (2.7), the operator is denoted by $\mathcal{H}_{0}^{D}$, in the case of condition (2.8), the notation is $\mathcal{H}_{0}^{R}$. As the domains of these operators, the following dense in $L_{2}\left(\Gamma_{0}\right)$ subsets serve:

$$
\begin{aligned}
& \mathfrak{D}\left(\mathcal{H}_{0}^{D}\right):=\left\{u=\left(u_{-}, u_{+}\right) \in L_{2}\left(\Gamma_{0}\right): u_{ \pm} \in W_{2}^{2}\left(e_{ \pm}\right) \text {, conditions 2.6, 2.7 hold }\right\}, \\
& \mathfrak{D}\left(\mathcal{H}_{0}^{R}\right):=\left\{u=\left(u_{-}, u_{+}\right) \in L_{2}\left(\Gamma_{0}\right): u_{ \pm} \in W_{2}^{2}\left(e_{ \pm}\right) \text {, conditions 2.6, 2.8 hold }\right\} .
\end{aligned}
$$

The operators $\mathcal{H}_{0}^{D}, \mathcal{H}_{0}^{R}$ are self-adjoint.

By $U_{ \pm}=U_{ \pm}\left(x_{ \pm}, \lambda\right)$ we denote the solutions to the Cauchy problems:

$$
-U_{ \pm}^{\prime \prime}+\left(W_{ \pm}-\lambda\right) U_{ \pm}=0 \quad \text { in } \quad\left(0, a_{ \pm}\right), \quad U_{ \pm}\left(a_{ \pm}, \lambda\right)=0, \quad U_{ \pm}^{\prime}\left(a_{ \pm}, \lambda\right)=1 .
$$

Such problems are uniquely solvable and their solutions are holomorphic in $\lambda \in \mathbb{C}$ in the norm $W_{2}^{2}\left(0, a_{ \pm}\right)$. For $\operatorname{Im} \lambda \neq 0$ we denote

$$
\Psi(x):=\left\{\begin{array}{lll}
\frac{U_{-}\left(x_{-}, \lambda\right)}{U_{-}(0, \lambda)} & \text { on } e_{-}, \\
\frac{U_{+}\left(x_{+}, \lambda\right)}{U_{+}(0, \lambda)} & \text { on } e_{+} .
\end{array}\right.
$$

This function is well-defined and $U_{ \pm}(0, \lambda) \neq 0$ as $\operatorname{Im} \lambda \neq 0$ since otherwise the Schrödinger operators $-\frac{d^{2}}{d x^{2}}+W_{ \pm}$on the edges $e_{ \pm}$with Dirichlet conditions would have possessed complex eigenvalues.

Our first result describes the uniform resolvent convergence of the operators $\mathcal{H}_{\varepsilon}^{D}$ and $\mathcal{H}_{\varepsilon}^{R}$ to the operators $\mathcal{H}_{0}^{D}$ and $\mathcal{H}_{0}^{R}$. A convergence theorem for the operator $\mathcal{H}_{\varepsilon}^{D}$ is as follows. 
Theorem 2.1. As $\operatorname{Im} \lambda \neq 0$, for each $f \in L_{2}\left(\Gamma_{\varepsilon}\right)$, the estimates hold:

$$
\begin{aligned}
& \left\|\left(\mathcal{H}_{\varepsilon}^{D}-\lambda\right)^{-1} f-\left.\left(\mathcal{H}_{0}^{D}-\lambda\right)^{-1} f\right|_{\Gamma_{0}}-\ell_{e}^{D}\left(f_{\varepsilon}\right) \Psi\right\|_{W_{2}^{2}\left(e_{-}\right) \oplus W_{2}^{2}\left(e_{+}\right)} \leqslant c \varepsilon^{\frac{5}{2}}\|f\|_{L_{2}\left(e_{\varepsilon}\right)}, \\
& \left\|\left(\mathcal{H}_{\varepsilon}^{D}-\lambda\right)^{-1} f\right\|_{L_{2}\left(e_{\varepsilon}\right)} \leqslant C \varepsilon\|f\|_{L_{2}\left(e_{\varepsilon}\right)},
\end{aligned}
$$

where $c$ is a constant independent of $f$ and $\varepsilon$,

$$
\ell_{\varepsilon}^{D}\left(f_{\varepsilon}\right):=\int_{0}^{\varepsilon} x_{\varepsilon} f_{\varepsilon}\left(x_{\varepsilon}\right) d x_{\varepsilon}, \quad\left|\ell_{\varepsilon}^{D}\left(f_{\varepsilon}\right)\right| \leqslant \frac{\varepsilon^{\frac{3}{2}}}{\sqrt{3}}\left\|f_{\varepsilon}\right\|_{L_{2}\left(e_{\varepsilon}\right)} .
$$

The results on convergence of the resolvents of the operator $\mathcal{H}_{\varepsilon}^{R}$ are provided in the next theorem.

Theorem 2.2. As $\operatorname{Im} \lambda \neq 0$, for each $f \in L_{2}\left(\Gamma_{\varepsilon}\right)$, the estimates hold:

$$
\begin{aligned}
& \left\|\left(\mathcal{H}_{\varepsilon}^{R}-\lambda\right)^{-1} f-\left.\left(\mathcal{H}_{0}^{R}-\lambda\right)^{-1} f\right|_{\Gamma_{0}}-\varepsilon \ell_{\varepsilon}^{R}(f) \Psi\right\|_{W_{2}^{2}\left(e_{-}\right) \oplus W_{2}^{2}\left(e_{+}\right)} \leqslant c \varepsilon^{\frac{5}{2}}\|f\|_{L_{2}\left(e_{\varepsilon}\right)}, \\
& \left\|\left(\mathcal{H}_{\varepsilon}^{R}-\lambda\right)^{-1} f\right\|_{L_{2}\left(e_{\varepsilon}\right)} \leqslant c \varepsilon\|f\|_{L_{2}\left(e_{\varepsilon}\right)},
\end{aligned}
$$

where $c$ is some constant independent of $f$ and $\varepsilon$,

$$
\ell_{\varepsilon}^{R}(f):=\frac{U_{-}(0, \lambda) U_{+}(0, \lambda)}{F(\lambda)-(\alpha+\beta) U_{-}(0, \lambda) U_{+}(0, \lambda)} \int_{0}^{\varepsilon} f\left(x_{\varepsilon}\right) d x_{\varepsilon}, \quad\left|\ell_{\varepsilon}^{R}(f)\right| \leqslant c \varepsilon^{\frac{1}{2}}\|f\|_{L_{2}\left(e_{\varepsilon}\right)} .
$$

The expression $F(\lambda)-(\alpha+\beta) U_{-}(0, \lambda) U_{+}(0, \lambda)$ is non-zero as $\operatorname{Im} \lambda \neq 0$.

The operators $\mathcal{H}_{\varepsilon}^{D}, \mathcal{H}_{\varepsilon}^{R}, \mathcal{H}_{0}^{D}$, and $\mathcal{H}_{0}^{R}$ have compact resolvents and their spectra are pure discrete. By $\lambda_{n}^{D}(\varepsilon)$ and $\lambda_{n}^{R}(\varepsilon)$ we denote the eigenvalues of the operators $\mathcal{H}_{\varepsilon}^{D}$ and $\mathcal{H}_{\varepsilon}^{R}$ taken in the ascending order counting the multiplicities. Our second result describes the behavior of these eigenvalues as $\varepsilon \rightarrow+0$. Before we formulate this result, let us describe the spectra of the operators $\mathcal{H}_{0}^{D}$ and $\mathcal{H}_{0}^{R}$.

The eigenvalues of the operator $\mathcal{H}_{0}^{D}$ coincide with the roots of the equation

$$
U_{-}(0, \lambda) U_{+}(0, \lambda)=0 \text {. }
$$

We denote these roots by $\Lambda_{n}^{D}, n \in \mathbb{N}$, and take them in ascending order counting the multiplicities as of eigenvalues. If some $\Lambda_{n}^{D}$ is a zero of only one of the functions $U_{ \pm}(0, \lambda)$, then such eigenvalue is simple. If it is a zero of both functions $U_{ \pm}(0, \lambda)$, then such eigenvalues is double and in this case $\Lambda_{n}^{D}=\Lambda_{n+1}^{D}$ according the chosen ordering.

Theorem 2.3. For sufficiently small $\varepsilon$, the eigenvalues $\lambda_{n}^{D}(\varepsilon)$ are holomorphic in $\varepsilon$ and $\lambda_{n}^{D}(0)=\Lambda_{n}^{D}$. If $\Lambda_{n}^{D}$ is a simple eigenvalue, then the eigenvalue $\lambda_{n}^{D}(\varepsilon)$ is also simple and

$$
\frac{d \lambda_{n}^{D}}{d \varepsilon}(0)=-\frac{\left(U_{ \pm}^{\prime}\left(0, \Lambda_{n}^{D}\right)\right)^{2}}{\left\|U_{ \pm}\left(\cdot, \Lambda_{n}^{D}\right)\right\|_{L_{2}\left(e_{ \pm}\right)}^{2}} \quad \text { as } \quad U_{ \pm}\left(0, \Lambda_{n}^{D}\right)=0, \quad U_{\mp}\left(0, \Lambda_{n}^{D}\right)=0 .
$$

If $\Lambda_{n}^{D}=\Lambda_{n+1}^{D}$ is a double eigenvalue, then the operator $\mathcal{H}_{\varepsilon}^{D}$ possesses two eigenvalues $\lambda_{n}^{D}(\varepsilon)$ and $\lambda_{n+1}^{D}(\varepsilon)$ converging to $\Lambda_{n}^{D}$ as $\varepsilon \rightarrow+0$. The eigenvalue $\lambda_{n}^{D}(\varepsilon)$ is holomorphic in $\varepsilon$ and the identity holds:

$$
\frac{d \lambda_{n}^{D}}{d \varepsilon}(0)=-\frac{\left(U_{-}^{\prime}\left(0, \Lambda_{n}^{D}\right)\right)^{2}}{\left\|U_{-}\left(\cdot, \Lambda_{n}^{-}\right)\right\|_{L_{2}\left(e_{-}\right)}^{2}}-\frac{\left(U_{+}^{\prime}\left(0, \Lambda_{n}^{D}\right)\right)^{2}}{U_{+}\left(\cdot, \Lambda_{n}^{+}\right) \|_{L_{2}\left(e_{+}\right)}^{2}} .
$$

The eigenvalue $\lambda_{n+1}^{D}(\varepsilon)$ is independent of $\varepsilon$ and coincides with $\Lambda_{n}^{D}$. 
We denote

$$
F(\lambda):=U_{+}^{\prime}(0, \lambda) U_{-}(0, \lambda)+U_{-}^{\prime}(0, \lambda) U_{+}(0, \lambda)
$$

The eigenvalues of the operator $\mathcal{H}_{0}^{R}$ coincide with the roots of the equation

$$
F(\lambda)-(\alpha+\beta) U_{-}(0, \lambda) U_{+}(0, \lambda)=0 .
$$

We denote these eigenvalues by $\Lambda_{n}^{R}$ and take them in the ascending order counting the multiplicities.

Theorem 2.4. All eigenvalues $\Lambda_{n}^{R}$ are simple. For each $\Lambda_{n}^{R}$, the numbers $U_{-} 0\left(0, \Lambda_{n}^{R}\right)$ are $U_{+} 0\left(0, \Lambda_{n}^{R}\right)$ vanish simultaneously.

For sufficiently small $\varepsilon$, the eigenvalues $\lambda_{n}^{R}(\varepsilon)$ are holomorphic in $\varepsilon$ and the identities hold $\lambda_{n}^{R}(0)=\Lambda_{n}^{R}$. If $U_{ \pm}\left(0, \Lambda_{n}^{R}\right)=0$, then $\lambda_{n}^{R}(\varepsilon)$ is independent of $\varepsilon$ and coincides with $\Lambda_{n}^{R}$. If $U_{ \pm}\left(0, \Lambda_{n}^{R}\right) \neq 0$, the identities hold:

$$
\frac{d \lambda_{n}^{R}}{d \varepsilon}(0)=\frac{U_{-}\left(0, \Lambda_{n}^{R}\right) U_{+}\left(0, \Lambda_{n}^{R}\right)\left(\int_{0}^{1} W_{0}(t) d t-\int_{0}^{1}\left(\int_{t}^{1} W_{-1}(s) d s\right)^{2} d t+\Lambda_{n}^{R}\right)}{\left(U_{+}\left(0, \Lambda_{n}^{R}\right)\right)^{2}\left\|U_{-}\left(\cdot, \Lambda_{n}^{-}\right)\right\|_{L_{2}\left(e_{-}\right)}^{2}+\left(U_{-}\left(0, \Lambda_{n}^{R}\right)\right)^{2}\left\|U_{+}\left(\cdot, \Lambda_{n}^{+}\right)\right\|_{L_{2}\left(e_{+}\right)}^{2}} .
$$

Let us discuss the main results. First of all we stress that despite a simple form of the graph $\Gamma_{\varepsilon}$, the obtained results are quite rich by their content. According Theorems 2.1, 2.2, as the the edge $e_{\varepsilon}$ shrinks, the resolvents of the operators $\mathcal{H}_{\varepsilon}^{D}$ and $\mathcal{H}_{\varepsilon}^{R}$ converge to the resolvents of the operators $\mathcal{H}_{0}^{D}$ and $\mathcal{H}_{0}^{R}$. For the operator $\mathcal{H}_{\varepsilon}^{D}$, the convergence means that if the boundary vertex of the small edge is subject to the Dirichlet condition, then in the limit $\varepsilon \rightarrow+0$, this boundary condition replaces the original Kirchoff condition in the internal vertex $M_{0}$. In view of work [8], this is a rather expectable result. At the same time, Theorem 2.1 provides more information on information about the convergence. Namely, estimate $(2.10)$ is established in a stronger norm of bounded operators acting from $L_{2}\left(\Gamma_{\varepsilon}\right)$ into $W_{2}^{2}\left(e_{-}\right) \oplus W_{2}^{2}\left(e_{+}\right)$. Moreover, in this estimate we also provide the first corrector in the expansion of the resolvent, which is the term $\ell_{e}^{D}\left(f_{\varepsilon}\right) \Psi$. It is small by 2.12). Estimate (2.11) means that the resolvent of the perturbed operator restricted on the small edge $e_{\varepsilon}$ is small in $L_{2}\left(e_{\varepsilon}\right)$-norm. Let us also pay a special attention to the right hand sides of estimates (2.10) and (2.11). They involve only the norm of the right hand side over the small edge. This means that if the right hand side vanishes on the small edge, then the actions of the resolvents of the operators $\mathcal{H}_{\varepsilon}^{D}$ and $\mathcal{H}_{0}^{D}$ on such functions $f$ coincides on $\Gamma_{0}$ and the actions of the resolvent of the operator $\mathcal{H}_{\varepsilon}^{D}$ on the small edge vanishes. In other words, the difference depends only on values of the right hand side on the small edge.

A similar situation holds for the operators $\mathcal{H}_{\varepsilon}^{R}$ and $\mathcal{H}_{0}^{R}$. Despite now on the boundary vertex of the edge $e_{\varepsilon}$ the Neumann condition is imposed, the limiting boundary value at the vertex $M_{0}$ involves a special coefficient $\beta$, see $(2.8)$. This coefficient is due to the presence of the potential $W_{-1}$ on the edge $e_{\varepsilon}$ in the perturbed operator. Such phenomenon agrees the well-known results on approximation of one-dimensional delta-interactions by potentials of form $\varepsilon^{-1} V\left(\frac{x}{\varepsilon}\right)$ with compactly supported functions $V$ having non-zero mean, see, for instance, [6, Ch. 1.3].

The presence of uniform resolvent convergence for the operators with singular perturbation is a rather expectable fact once we compare the operators on graphs with those in multidimensional domains. As an example, we mention problems in the domains with small holes, a classical model in the theory of singular perturbations. A uniform resolvent convergence for such problems was proved in works [1], [2]. From this point of view, singularly perturbed operators exhibit properties similar to regularly perturbed operators. At the same time, in the case of regular perturbations, the resolvents and eigenvalues are holomorphic in a small parameter describing the perturbation. As a rule, the same statement fails for singularly perturbed operators. For instance, in the classical problem in domains with small holes, one can construct complete asymptotic expansions in the small parameter, [3, Ch. III], [4], but 
no statements on convergence of these series and moreover, on their sums coinciding with the perturbed resolvents and eigenvalues, were proved.

In view of the said above, the results of Theorems 2.3, 2.4 look quite unexpectable and interesting. The main key statement of these theorems is on the holomorphic dependence of the eigenvalues on the small parameter. In other words, the asymptotic series of the eigenvalues of the considered operators converge and the sums coincide with the perturbed eigenvalues. In addition, we find one more phenomenon, stable eigenvalues. For the operators $\mathcal{H}_{\varepsilon}^{D}$, such eigenvalues arise in the vicinity of limiting double eigenvalue: each such limiting eigenvalue splits into two perturbed eigenvalues, one of which is stable and independent of $\varepsilon$, while the other moves to the left. For the operator $\mathcal{H}_{\varepsilon}^{R}$ a similar picture holds as well if the eigenfunction corresponding to the limiting eigenvalue vanishes at the vertex $M_{0}$. Here the limiting eigenvalue is simple but is stable under the perturbation.

For moving eigenvalues of the perturbed operators, we find their first correctors in Theorems 2.3, 2.4, see formulae 2.17), 2.19). As it follows from formula (2.17), in the case of the Dirichlet condition at the vertex $M_{\varepsilon}$, the first correctors are negative and adding a small edge acts as a non-positive perturbation. In the case of the Neumann condition at the vertex $M_{\varepsilon}$, the first corrector becomes more complicated and apriori its sign is unclear.

We expect that the described phenomena are not due to a simple structure of graphs the $\Gamma_{\varepsilon}$ and $\Gamma_{0}$ but a general feature of a wide class of graphs with small edges. This conjecture will be justified in one of our future works.

\section{Asymptotics For Resolvent of operator $\mathcal{H}_{\varepsilon}^{D}$}

In the present section we prove Theorem 2.1. Given $f \in L_{2}\left(\Gamma_{\varepsilon}\right)$, by $f_{0}$ we denote the restriction of $f$ on the graph $\Gamma_{0}$, while $f_{\varepsilon}$ stands for the restriction of $f$ on the edge $e_{\varepsilon}$. We let $u_{\varepsilon}:=\left(\mathcal{H}_{\varepsilon}^{D}-\lambda\right)^{-1} f, u_{0}:=\left(\mathcal{H}_{0}^{D}-\lambda\right)^{-1} f_{0}$. In view of boundary condition (2.2) and the definition of functions $U_{ \pm}$, it is clear that

$$
u_{\varepsilon}=u_{0}+C_{\varepsilon} \Psi \quad \text { on } \quad \Gamma_{0},
$$

where $C_{\varepsilon}$ is some constant, which will be determined later.

To determine the function $u_{\varepsilon}$ on the edge $e_{\varepsilon}$, we consider an auxiliary Cauchy problem

$$
-U_{D}^{\prime \prime}+\left(\varepsilon W_{-1}(\xi)+\varepsilon^{2} W_{0}(\xi)-\mu\right) U_{D}=0, \quad \xi \in(0,1),\left.\quad U_{D}\right|_{\xi=1}=0,\left.\quad U_{D}^{\prime}\right|_{\xi=1}=1
$$

where $\mu$ is a small complex parameter. This problem is uniquely solvable, possesses a solution $U_{D}=U_{D}(\xi, \varepsilon, \mu)$ holomorphic with respect to $\varepsilon$ and $\mu$ in $W_{2}^{2}(0,1)$-norm. By straightforward calculations we confirm that

$$
U_{D}(\xi, \varepsilon, \mu)=\xi-1+O(\varepsilon+|\mu|) .
$$

By $\mathcal{S}_{\varepsilon}^{D}$ we denote the Schrödinger operator in the space $L_{2}(0,1)$ with the differential expression

$$
-\frac{d^{2}}{d \xi^{2}}+\varepsilon W_{-1}(\xi)+\varepsilon^{2} W_{0}(\xi)
$$

subject to the Dirichlet condition. The domain of the operator $\mathcal{S}_{\varepsilon}^{D}$ is the set of the functions in $W_{2}^{2}(0,1)$ vanishing at the boundary. It is clear that the operator $\mathcal{S}_{0}^{D}$ is invertible and the inverse is bounded as an operator from $L_{2}(0,1)$ into $W_{2}^{2}(0,1)$. This is why the same is true for the operator $\mathcal{S}_{\varepsilon}^{D}-\varepsilon^{2} \lambda$ : for sufficiently small $\varepsilon$, the inverse operator $\left(\mathcal{S}_{\varepsilon}^{D}-\varepsilon^{2} \lambda\right)^{-1}$ is well-defined as a bounded operator from $L_{2}(0,1)$ into $W_{2}^{2}(0,1)$ and it is also holomorphic in $\varepsilon$. In particular,

$$
\left(\mathcal{S}_{\varepsilon}^{D}-\varepsilon^{\lambda}\right)^{-1}=\left(\mathcal{S}_{0}^{D}\right)^{-1}+O(\varepsilon), \quad\left(\left(\mathcal{S}_{0}^{D}\right)^{-1} g\right)(\xi)=-\int_{0}^{1} \frac{|\xi-t|-\xi-t+2 \xi t}{2} g(t) d t
$$


where $g \in L_{2}(0,1)$. In view of the first boundary condition in (2.2) and formula (3.1), it is easy to see that on the edge $e_{\varepsilon}$, the function $u_{\varepsilon}$ reads as

$$
u_{\varepsilon}\left(x_{\varepsilon}\right)=\varepsilon^{2} v_{\varepsilon}\left(\frac{x_{\varepsilon}}{\varepsilon}\right)+C_{\varepsilon} \frac{U_{D}\left(\frac{x_{\varepsilon}}{\varepsilon}, \varepsilon, \varepsilon^{2} \lambda\right)}{U_{D}\left(0, \varepsilon, \varepsilon^{2} \lambda\right)}, \quad v_{\varepsilon}:=\left(\mathcal{S}_{\varepsilon}^{D}-\varepsilon^{2} \lambda\right)^{-1} f_{\varepsilon}(\varepsilon \cdot) .
$$

We determine the constant $C_{\varepsilon}$ by the second condition in $(2.2)$ and identities (3.1), (3.6):

$$
C_{\varepsilon}\left(\frac{U_{-}^{\prime}(0, \lambda)}{U_{-}(0, \lambda)}+\frac{U_{+}^{\prime}(0, \lambda)}{U_{+}(0, \lambda)}-\alpha+\varepsilon^{-1} \frac{U_{D}^{\prime}\left(0, \varepsilon, \varepsilon^{2} \lambda\right)}{U_{D}\left(0, \varepsilon, \varepsilon^{2} \lambda\right)}\right)+\varepsilon v_{\varepsilon}^{\prime}(0)=0 .
$$

According identities $(3.3)$, for small $\varepsilon$ the relation holds:

$$
\frac{U_{D}^{\prime}\left(0, \varepsilon, \varepsilon^{2} \lambda\right)}{U_{D}\left(0, \varepsilon, \varepsilon^{2} \lambda\right)}=1+O(\varepsilon) \neq 0
$$

and this is why by equation 3.7 we can determine the constant $C_{\varepsilon}$ :

$$
C_{\varepsilon}:=-\frac{\varepsilon^{2} v_{\varepsilon}^{\prime}(0)}{\frac{U_{D}^{\prime}\left(0, \varepsilon, \varepsilon^{2} \lambda\right)}{U_{D}\left(0, \varepsilon, \varepsilon^{2} \lambda\right)}+\varepsilon\left(\frac{U_{-}^{\prime}(0, \lambda)}{U_{-}(0, \lambda)}+\frac{U_{+}^{\prime}(0, \lambda)}{U_{+}(0, \lambda)}-\alpha\right)} .
$$

It follows from relations $(3.5)$ that

$$
\left|v_{\varepsilon}^{\prime}(0)-\varepsilon^{-1} \int_{0}^{\varepsilon}\left(1-\frac{x_{\varepsilon}}{\varepsilon}\right) f_{\varepsilon}\left(x_{\varepsilon}\right) d x_{\varepsilon}\right| \leqslant c \varepsilon\left\|f_{\varepsilon}(\varepsilon \cdot)\right\|_{L_{2}(0,1)}=c \varepsilon^{\frac{1}{2}}\left\|f_{\varepsilon}\right\|_{L_{2}\left(e_{\varepsilon}\right)} .
$$

Hereinafter the symbol $c$ stands for various inessential constants independent of $\varepsilon$ and $f$. We also observe that by the Cauchy-Schwarz inequality, the estimate

$$
\left|\varepsilon^{2} \int_{0}^{\varepsilon} f\left(x_{\varepsilon}\right) d x_{\varepsilon}\right| \leqslant \varepsilon^{\frac{5}{2}}\left\|f_{\varepsilon}\right\|_{L_{2}\left(\Gamma_{\varepsilon}\right)}, \quad\left|\int_{0}^{\varepsilon} x_{\varepsilon} f\left(x_{\varepsilon}\right) d x_{\varepsilon}\right| \leqslant \frac{\varepsilon^{\frac{3}{2}}}{\sqrt{3}}\left\|f_{\varepsilon}\right\|_{L_{2}\left(\Gamma_{\varepsilon}\right)}
$$

holds true. We substitute the obtained estimate, (3.8), (3.10) in $(3.9)$ to obtain

$$
\left|C_{\varepsilon}-\int_{0}^{\varepsilon} x_{\varepsilon} f\left(x_{\varepsilon}\right) d x_{\varepsilon}\right| \leqslant c \varepsilon^{\frac{5}{2}}\left\|f_{\varepsilon}\right\|_{L_{2}\left(e_{\varepsilon}\right)} .
$$

Inequality 2.10 follows the above estimate and formula (3.1).

Let us prove estimate (2.11). First we observe that it follows from $(3.12)$ and the second inequality in (3.11) that

$$
\left|C_{\varepsilon}\right| \leqslant c \varepsilon^{\frac{3}{2}}\left\|f_{\varepsilon}\right\|_{L_{2}\left(e_{\varepsilon}\right)} .
$$

We can estimate the norm of the function $-v_{\varepsilon}$ by the aforementioned properties of the operator $\left(\mathcal{S}_{\varepsilon}^{D}-\varepsilon^{2} \lambda\right)^{-1}$ :

$$
\left\|v_{\varepsilon}\left(\frac{\dot{-}}{\varepsilon}\right)\right\|_{L_{2}\left(e_{\varepsilon}\right)}=\varepsilon^{-\frac{1}{2}}\left\|v_{\varepsilon}\right\|_{L_{2}(0,1)} \leqslant c \varepsilon^{-\frac{1}{2}}\left\|f_{\varepsilon}(\varepsilon \cdot)\right\|_{L_{2}(0,1)}=c \varepsilon^{-1}\left\|f_{\varepsilon}\right\|_{L_{2}\left(e_{\varepsilon}\right)} .
$$

This yields:

$$
\left\|\varepsilon^{2} v_{\varepsilon}\left(\frac{\dot{-}}{\varepsilon}\right)\right\|_{L_{2}\left(e_{\varepsilon}\right)} \leqslant c \varepsilon\left\|f_{\varepsilon}\right\|_{L_{2}\left(e_{\varepsilon}\right)}
$$

Taking into consideration the obvious relations

$$
\left\|U_{D}\left(\frac{\dot{\varepsilon}}{\varepsilon}, \varepsilon, \varepsilon^{2} \lambda\right)\right\|_{L_{2}\left(e_{\varepsilon}\right)}=\varepsilon^{\frac{1}{2}}\left\|U_{D}\left(\cdot, \varepsilon, \varepsilon^{2} \lambda\right)\right\|_{L_{2}(0,1)} \leqslant c \varepsilon^{\frac{1}{2}}
$$

by (3.13) and (3.6) we get estimate 2.11). The proof of Theorem 2.1 is complete. 


\section{Asymptotics For RESOlVEnt OF OPERATOR $\mathcal{H}_{\varepsilon}^{R}$}

Here we prove Theorem 2.2. As in the previous section, by $f_{0}$ we denote the restriction of $f$ on the graph $\Gamma_{0}$, while $f_{\varepsilon}$ is the restriction of $f$ on the edge $e_{\varepsilon}$. We let $u_{\varepsilon}:=\left(\mathcal{H}_{\varepsilon}^{R}-\lambda\right)^{-1} f$, $u_{0}:=\left(\mathcal{H}_{0}^{D}-\lambda\right)^{-1} f_{\varepsilon}$. The latter functions again obey identity 3.1) with some constant $C_{\varepsilon}$. Instead of Cauchy problem (3.2), here we consider the following one:

$$
-U_{R}^{\prime \prime}+\left(\varepsilon W_{-1}(\xi)+\varepsilon^{2} W_{0}(\xi)-\mu\right) U_{R}=0, \quad \xi \in(0,1),\left.\quad U_{R}\right|_{\xi=1}=1,\left.\quad U_{R}^{\prime}\right|_{\xi=1}=0,
$$

where $\mu$ is a small complex parameter. This problem is also uniquely solvable and its solution $U_{R}=U_{R}(\xi, \varepsilon, \mu)$ is holomorphic in $\varepsilon$ and $\mu$ in the norm of $W_{2}^{2}(0,1)$. It is easy to confirm that the leading terms of the expansion of the function $U_{R}$ is of the form:

$$
\begin{aligned}
& U_{R}(\xi, \varepsilon, \mu)=1+\varepsilon \phi_{1}(\xi)+\varepsilon^{2} \phi_{2}(\xi)+\mu \phi_{3}(\xi)+O\left(\varepsilon^{3}+\varepsilon|\mu|+|\mu|^{2}\right), \\
& \phi_{1}(\xi)=\int_{\xi}^{1}(t-\xi) W_{-1}(t) d t, \quad \phi_{3}(\xi)=\frac{(1-\xi)^{2}}{2}, \\
& \phi_{2}(\xi)=\int_{\xi}^{1}(t-\xi)\left(W_{0}(t)+W_{-1}(t) \phi_{1}(t)\right) d t .
\end{aligned}
$$

We also observe that the number $\beta$ should satisfy the identity

$$
\beta=-\phi_{1}^{\prime}(0) .
$$

Instead of the operator $\mathcal{S}_{\varepsilon}^{D}$, we should take the Schrödinger operator in $L_{2}(0,1)$ with differential expression (3.4), the Dirichlet condition at the point $\xi=0$ and the Neumann condition at the point $\xi=1$. We denote such operator by $\mathcal{S}_{\varepsilon}^{R}$. It possesses the same properties as the operator $\mathcal{S}_{\varepsilon}^{D}$, namely, for sufficiently small $\varepsilon$, the inverse operator $\left(\mathcal{S}_{\varepsilon}^{R}-\varepsilon^{2} \lambda\right)^{-1}$ is well-defined, bounded as an operator from $L_{2}(0,1)$ into $W_{2}^{2}(0,1)$, holomorphic in $\varepsilon$ and the relations hold:

$$
\left(\mathcal{S}_{\varepsilon}^{R}-\varepsilon^{\lambda}\right)^{-1}=\left(\mathcal{S}_{0}^{R}\right)^{-1}+O(\varepsilon), \quad\left(\left(\mathcal{S}^{R}\right)^{-1} g\right)(\xi)=-\int_{0}^{1} \frac{|\xi-t|-\xi-t}{2} g(t) d t
$$

where $g \in L_{2}(0,1)$. An analogue of identity (3.6) reads as follows:

$$
u_{\varepsilon}(x)=\varepsilon^{2} v_{\varepsilon}\left(\frac{x_{\varepsilon}}{\varepsilon}\right)+C_{\varepsilon} \frac{U_{R}\left(\frac{x_{\varepsilon}}{\varepsilon}, \varepsilon, \varepsilon^{2} \lambda\right)}{U_{R}\left(0, \varepsilon, \varepsilon^{2} \lambda\right)}, \quad v_{\varepsilon}:=\left(\mathcal{S}_{\varepsilon}^{R}-\varepsilon^{2} \lambda\right)^{-1} f_{\varepsilon}(\varepsilon \cdot)
$$

this ensures the first condition in (2.2). Substituting (3.1), (4.4) into the second condition gives the equation for $C_{\varepsilon}$ :

$$
C_{\varepsilon}\left(\frac{U_{-}^{\prime}(0, \lambda)}{U_{-}(0, \lambda)}+\frac{U_{+}^{\prime}(0, \lambda)}{U_{+}(0, \lambda)}-\alpha+\varepsilon^{-1} \frac{U_{R}^{\prime}\left(0, \varepsilon, \varepsilon^{2} \lambda\right)}{U_{R}\left(0, \varepsilon, \varepsilon^{2} \lambda\right)}\right)+\varepsilon v_{\varepsilon}^{\prime}(0)=0 .
$$

According 4.1), 4.2), for small $\varepsilon$ the identities hold true:

$$
\varepsilon^{-1} \frac{U_{R}^{\prime}\left(0, \varepsilon, \varepsilon^{2} \lambda\right)}{U_{R}\left(0, \varepsilon, \varepsilon^{2} \lambda\right)}=-\beta+O(\varepsilon)
$$

We also observe that

$$
\frac{U_{-}^{\prime}(0, \lambda)}{U_{-}(0, \lambda)}+\frac{U_{+}^{\prime}(0, \lambda)}{U_{+}(0, \lambda)}-\alpha-\beta=\frac{F(\lambda)-(\alpha+\beta) U_{-}(0, \lambda) U_{+}(0, \lambda)}{U_{-}(0, \lambda) U_{+}(0, \lambda)} \neq 0,
$$


since otherwise $\lambda$ is a complex eigenvalue of the operator $\mathcal{H}_{0}^{R}$ with the corresponding eigenfunction equalling to $\frac{U_{ \pm}\left(x_{ \pm}, \lambda\right)}{U_{ \pm}(0, \lambda)}$ on $e_{ \pm}$. The constant $C_{\varepsilon}$ is found by the formula:

$$
C_{\varepsilon}=-\frac{\varepsilon v_{\varepsilon}^{\prime}(0)}{\frac{U_{-}^{\prime}(0, \lambda)}{U_{-}(0, \lambda)}+\frac{U_{+}^{\prime}(0, \lambda)}{U_{+}(0, \lambda)}-\alpha-\beta+\left(\varepsilon^{-1} \frac{U_{R}^{\prime}\left(0, \varepsilon, \varepsilon^{2} \lambda\right)}{U_{R}\left(0, \varepsilon, \varepsilon^{2} \lambda\right)}+\beta\right)},
$$

and the second term in the denominator is of order $O(\varepsilon)$ by 4.5$)$.

Let us find out the behavior of the numerator in (4.7). Employing (4.3), by straightforward calculations we check that

$$
\left|v_{\varepsilon}^{\prime}(0)-\int_{0}^{1} f_{\varepsilon}(\varepsilon t) d t\right| \leqslant c \varepsilon\left\|f_{\varepsilon}(\varepsilon \cdot)\right\|_{L_{2}(0,1)}=c \varepsilon^{\frac{1}{2}}\left\|f_{\varepsilon}\right\|_{L_{2}\left(e_{\varepsilon}\right)}, \quad \int_{0}^{1} f_{\varepsilon}(\varepsilon t) d t=\varepsilon^{-1} \int_{0}^{\varepsilon} f_{\varepsilon}\left(x_{\varepsilon}\right) d x_{\varepsilon} .
$$

Substituting the obtained relations into (4.7) and bearing in mind (4.5), (4.6), we obtain:

$$
\left|C_{\varepsilon}+\frac{U_{-}(0, \lambda) U_{+}(0, \lambda)}{F(\lambda)-(\alpha+\beta) U_{-}(0, \lambda) U_{+}(0, \lambda)}\right| \leqslant c \varepsilon^{\frac{5}{2}}\left\|f_{\varepsilon}\right\|_{L_{2}\left(e_{\varepsilon}\right)} .
$$

By (3.1) this implies estimate (2.13).

It also follows from 4.8 that

$$
\left|C_{\varepsilon}\right| \leqslant c \varepsilon^{\frac{3}{2}}\left\|f_{\varepsilon}\right\|_{L_{2}\left(e_{\varepsilon}\right)}
$$

The first term in (4.4) obeys an estimate similar to (3.14), while the function $U_{R}$ satisfies an estimate similar to (3.15). These estimates and (4.9) prove (2.14).

\section{Spectrum of Operator $\mathcal{H}_{\varepsilon}^{D}$}

In the present section we find the spectrum of the operator $\mathcal{H}_{\varepsilon}^{D}$ and we prove Theorem 2.3.

The eigenvalues of the operator $\mathcal{H}_{0}^{D}$ are determined by equation 2.15 that follows boundary condition 2.7) written for the functions $U_{ \pm}$. The eigenfunctions are of the form:

$$
\Psi_{n}^{D}(x)=\left\{\begin{array}{ccc}
U_{-}\left(x_{-}, \Lambda_{n}^{D}\right) & \text { on } & e_{-}, \\
0 & \text { on } & e_{+}
\end{array}\right.
$$

if $\Lambda_{n}^{D}$ is a zero of the function $U_{-}(0, \lambda)$ and

$$
\Psi_{n}^{D}(x)=\left\{\begin{array}{ccc}
0 & \text { on } & e_{-}, \\
U_{+}\left(x_{+}, \Lambda_{n}^{D}\right) & \text { on } & e_{+}
\end{array}\right.
$$

if $\Lambda_{n}^{D}$ is a zero of the function $U_{-}(0, \lambda)$. If $\Lambda_{n}^{D}$ is a joint zero of the functions $U_{ \pm}(0, \lambda)$, then such eigenvalue is double with a pair of associated eigenfunctions determined by identities (5.1), 5.2 .

In what follows we make use of the following auxiliary lemma.

Lemma 5.1. The relations hold:

$$
\frac{d U_{ \pm}}{d \lambda}\left(0, \Lambda_{n}^{D}\right)=-\frac{1}{U_{ \pm}^{\prime}\left(0, \Lambda_{n}^{D}\right)}\left\|U_{ \pm}\left(\cdot, \Lambda_{n}^{D}\right)\right\|_{L_{2}\left(e_{ \pm}\right)}^{2} \neq 0, \quad U_{ \pm}^{\prime}\left(0, \Lambda_{n}^{D}\right) \neq 0 .
$$

Proof. Inequality $U_{ \pm}^{\prime}\left(0, \Lambda_{n}\right) \neq 0$ is obvious since otherwise we are led to the identity $U_{ \pm}\left(x, \Lambda_{n}\right)=$ 0 , which is false.

Differentiating problem (2.9) in $\lambda$, it is easy to infer that the functions

$$
v_{ \pm}\left(x_{ \pm}\right):=\frac{d U_{ \pm}}{d \lambda}\left(x_{ \pm}, \Lambda_{n}\right)
$$

solve the Cauchy problem

$$
-v_{ \pm}^{\prime \prime}+\left(V_{ \pm}-\Lambda_{n}^{D}\right) v_{ \pm}=U_{ \pm}\left(\cdot, \Lambda_{n}\right) \quad \text { in } \quad\left(0, a_{ \pm}\right), \quad v_{ \pm}\left(a_{ \pm}\right)=0, \quad v_{ \pm}^{\prime}\left(a_{ \pm}\right)=0 .
$$


Multiplying the equation in this problem by $U_{ \pm}\left(x_{ \pm}, \Lambda_{n}\right)$ and integrating twice by parts over the segment $\left(0, a_{ \pm}\right)$, we obtain the required formula.

Let us find the spectrum of the operator $\mathcal{H}_{\varepsilon}^{D}$. We construct the eigenfunctions of the operator $\mathcal{H}_{\varepsilon}^{D}$ as

$$
\Psi_{\varepsilon}^{D}(x):= \begin{cases}C_{-} U_{-}\left(x_{-}, \lambda\right) & \text { on } e_{-}, \\ C_{+} U_{+}\left(x_{+}, \lambda\right) & \text { on } e_{+}, \\ C_{\varepsilon} \varepsilon U_{D}\left(\frac{x_{\varepsilon}}{\varepsilon}, \varepsilon, \varepsilon^{2} \lambda\right) & \text { on } e_{\varepsilon}\end{cases}
$$

where $C_{ \pm}, C_{\varepsilon}$ are some constants. It is clear that such function satisfies the required differential equation and conditions (2.3). This is why it remains to check the condition at the vertex $M_{0}$ :

$$
\left\{\begin{array}{l}
C_{-} U_{-}(0, \lambda)-C_{+} U_{+}(0, \lambda)=0 \\
C_{-} U_{-}(0, \lambda)-C_{\varepsilon} \varepsilon U_{D}\left(0, \varepsilon, \varepsilon^{2} \mu\right)=0 \\
C_{-}\left(U_{-}^{\prime}(0, \lambda)+\alpha U_{-}(0, \lambda)\right)+C_{+} U_{+}^{\prime}(0, \lambda)+C_{\varepsilon} U_{D}^{\prime}\left(0, \varepsilon, \varepsilon^{2} \lambda\right)=0 .
\end{array}\right.
$$

The eigenvalues of the operator $\mathcal{H}_{\varepsilon}^{D}$ correspond to nontrivial solutions $\left(C_{-}, C_{+}, C_{\varepsilon}\right)$ of this system of linear equations. Applying the Cramer's rule to this system, we arrive at the equation for the eigenvalues:

$$
G^{D}(\lambda, \varepsilon)=0
$$

where

$$
G^{D}(\lambda, \varepsilon):=\varepsilon U_{D}\left(0, \varepsilon, \varepsilon^{2} \lambda\right) F(\lambda)+\left(U_{D}^{\prime}\left(0, \varepsilon, \varepsilon^{2} \lambda\right)-\varepsilon \alpha U_{D}\left(0, \varepsilon, \varepsilon^{2} \lambda\right)\right) U_{-}(0, \lambda) U_{+}(0, \lambda) .
$$

The function $G^{D}$ is holomorphic in $\lambda$ and $\varepsilon$. As $\varepsilon=0, \Lambda=\Lambda_{n}^{D}$, equation (5.3) obviously holds. By the holomorphic property of the functions $G^{D}$ in $\lambda$ and $\varepsilon$, we obtain immediately that the roots of equation $(5.3)$ converge to $\Lambda_{n}^{D}$ as $\varepsilon \rightarrow+0$.

To describe the behavior of roots of equation (5.3) for small $\varepsilon$, we need to study the structure of the function $G^{D}$ as $\lambda$ is close to $\Lambda_{n}^{D}$.

First we suppose that $\Lambda_{n}^{D}$ is a simple eigenvalue. For the sake of definiteness, we suppose that $\Lambda_{n}^{D}$ is a zero of the function $U_{-}(0, \lambda)$ and $U_{+}\left(0, \Lambda_{n}^{D}\right) \neq 0$. Then employing Lemma 5.1 and identities (3.3), it is straightforward to infer that

$$
\frac{d G^{D}}{d \lambda}\left(\Lambda_{n}^{D}, 0\right)=U_{+}\left(0, \Lambda_{n}^{D}\right) \frac{d U_{-}}{d \lambda}\left(0, \Lambda_{n}^{D}\right)=-\frac{U_{+}\left(0, \Lambda_{n}^{D}\right)}{U_{-}^{\prime}\left(0, \Lambda_{n}^{D}\right)}\left\|U_{-}\left(\cdot, \Lambda_{n}^{D}\right)\right\|_{L_{2}\left(e_{-}\right)}^{2} \neq 0 .
$$

Therefore, by the implicit function theorem, there exists the unique root $\lambda_{n}(\varepsilon)$ of equation (5.3) converging to $\Lambda_{n}^{D}$ and this root is holomorphic in $\varepsilon$. Since by identity 3.3 we have

$$
\frac{d G^{D}}{d \varepsilon}\left(\Lambda_{n}^{D}, 0\right)=-U_{-}^{\prime}\left(0, \Lambda_{n}^{D}\right) U_{+}\left(0, \Lambda_{n}^{D}\right)
$$

by (5.4) and the formula

$$
\frac{d \lambda_{n}^{D}}{d \varepsilon}(0)=-\frac{\frac{d G^{D}}{d \varepsilon}\left(\Lambda_{n}^{D}, 0\right)}{\frac{d G}{d \lambda}\left(\Lambda_{n}^{D}, 0\right)}
$$

we obtain immediately identity $(2.16)$.

Let $\Lambda_{n}^{D}$ be a double eigenvalue, that is, $\Lambda_{n}^{D}$ is a joint zero of the functions $U_{-}(0, \lambda)$ and $U_{+}(0, \lambda)$. Then it follows from the definition of the function $F$ and Lemma 5.1 that

$$
F(\lambda)=\left(\lambda-\Lambda_{n}^{D}\right) F_{*}(\lambda), \quad U_{+}(0, \lambda) U_{-}(0, \lambda)=\left(\lambda-\Lambda_{n}^{D}\right)^{2} Q(\lambda),
$$


where $F_{*}(\lambda), Q(\lambda)$ are holomorhpic functions and

$$
\begin{aligned}
& F_{*}\left(\Lambda_{n}^{D}\right)=-\frac{U_{+}^{\prime}\left(0, \Lambda_{n}^{D}\right)}{U_{-}^{\prime}\left(0, \Lambda_{n}^{D}\right)}\left\|U_{-}\left(\cdot, \Lambda_{n}^{D}\right)\right\|_{L_{2}\left(e_{-}\right)}^{2}-\frac{U_{-}^{\prime}\left(0, \Lambda_{n}^{D}\right)}{U_{+}^{\prime}\left(0, \Lambda_{n}^{D}\right)}\left\|U_{+}\left(\cdot, \Lambda_{n}^{D}\right)\right\|_{L_{2}\left(e_{+}\right)}^{2}, \\
& Q\left(\Lambda_{n}^{D}\right)=\frac{1}{U_{-}^{\prime}\left(0, \Lambda_{n}^{D}\right) U_{+}^{\prime}\left(0, \Lambda_{n}^{D}\right)}\left\|U_{-}\left(\cdot, \Lambda_{n}^{D}\right)\right\|_{L_{2}\left(e_{-}\right)}^{2}\left\|U_{+}\left(\cdot, \Lambda_{n}^{D}\right)\right\|_{L_{2}\left(e_{+}\right)}^{2} \neq 0 .
\end{aligned}
$$

Substituting relations (5.6), (5.7) into equation (5.3), we see that it has two roots converging to $\Lambda_{n}^{D}$. The first root is independent of $\varepsilon$ and coincides with $\Lambda_{n}^{D}$. The second root is determined by the equation

$$
\varepsilon U_{D}\left(0, \varepsilon, \varepsilon^{2} \lambda\right) F_{*}(\lambda)+\left(U_{D}^{\prime}\left(0, \varepsilon, \varepsilon^{2} \lambda\right)-\varepsilon \alpha U_{D}\left(0, \varepsilon, \varepsilon^{2} \lambda\right)\right)\left(\lambda-\Lambda_{n}^{D}\right) Q(\lambda)=0 .
$$

We apply the implicit function theorem to this equation in the same way as above. This immediately leads us to the conclusion that the latter equation possesses exactly one solution converging to $\Lambda_{n}^{D}$ as $\varepsilon \rightarrow+0$, this solution is holomorphic in $\varepsilon$ and identity 2.17$)$ holds. Since the left hands side of this identity is negative, it is cleat that for small positive $\varepsilon$ the root obeying 2.17) is less than $\Lambda_{n}^{D}$. The proof of Theorem 2.1 is complete.

\section{Spectrum of operator $\mathcal{H}_{\varepsilon}^{R}$}

The present section is devoted to the proof of Theorem 2.4. First we clarify the origination of equation (2.18). The eigenfunctions of the operator $\mathcal{H}_{0}^{R}$ are to be sough as

$$
\Psi^{R}(x):=\left\{\begin{array}{lll}
C_{-} U_{-}\left(x_{-}, \lambda\right) & \text { on } & e_{-}, \\
C_{+} U_{+}\left(x_{+}, \lambda\right) & \text { on } & e_{+}
\end{array}\right.
$$

where $C_{ \pm}$are some constants. Condition (2.8) leads us to a system of linear equations for these constants and the Cramer's rule allows us to find the cases when the system possesses a non-trivial solution. The vanishing of the corresponding determinant gives rise to equation (2.18).

Each eigenvalue $\Lambda_{n}^{R}$ is simple since otherwise we are led to the existence of an eigenfunction given by formula (6.1) with $C_{1}=0$. By boundary condition (2.8) this yields $C_{2}=0$ that contradicts the definition of an eigenfunction.

Assume now that $U_{-}\left(0, \Lambda_{n}^{R}\right)=0$. Then we necessarily have $U_{-}^{\prime}\left(0, \Lambda_{n}^{R}\right) \neq 0$ and it follows from equation (2.18) that $U_{+}\left(0, \Lambda_{n}^{R}\right)=0$. In the same way we check that the identity $U_{+}\left(0, \Lambda_{n}^{R}\right)=0$ implies $U_{-}\left(0, \Lambda_{n}^{R}\right)=0$. This is why the numbers $U_{-} 0\left(0, \Lambda_{n}^{R}\right)$ and $U_{+} 0\left(0, \Lambda_{n}^{R}\right)$ vanish simultaneously.

In what follows, we shall need an analogue of auxiliary lemma 5.1 .

Lemma 6.1. Let $U_{ \pm}\left(0, \Lambda_{n}^{R}\right)=0$. Then

$$
\frac{d F}{d \lambda}\left(\Lambda_{n}^{R}\right)=-\frac{U_{+}^{\prime}\left(0, \Lambda_{n}^{R}\right)}{U_{-}^{\prime}\left(0, \Lambda_{n}^{R}\right)}\left\|U_{-}\left(\cdot, \Lambda_{n}^{R}\right)\right\|_{L_{2}\left(e_{-}\right)}^{2}-\frac{U_{-}^{\prime}\left(0, \Lambda_{n}^{R}\right)}{U_{+}^{\prime}\left(0, \Lambda_{n}^{R}\right)}\left\|U_{+}\left(\cdot, \Lambda_{n}^{R}\right)\right\|_{L_{2}\left(e_{+}\right)}^{2} \neq 0 .
$$

Let $U_{ \pm}\left(0, \Lambda_{n}^{R}\right) \neq 0$. Then

$$
\begin{aligned}
\frac{d\left(F-(\alpha+\beta) U_{-}(0, \cdot) U_{+}(0, \cdot)\right)}{d \lambda}\left(\Lambda_{n}^{R}\right)= & \frac{U_{+}\left(0, \Lambda_{n}^{R}\right)}{U_{-}\left(0, \Lambda_{n}^{R}\right)}\left\|U_{-}\left(\cdot, \Lambda_{n}^{R}\right)\right\|_{L_{2}\left(e_{-}\right)}^{2} \\
& +\frac{U_{-}\left(0, \Lambda_{n}^{R}\right)}{U_{+}\left(0, \Lambda_{n}^{R}\right)}\left\|U_{+}\left(\cdot, \Lambda_{n}^{R}\right)\right\|_{L_{2}\left(e_{+}\right)}^{2} \neq 0 .
\end{aligned}
$$

Proof. Similar to the proof of Lemma 5.1, it is easy to check the inequalities:

$$
U_{ \pm}\left(0, \Lambda_{n}^{R}\right) \frac{d U_{ \pm}^{\prime}}{d \lambda}\left(0, \Lambda_{n}^{R}\right)-U_{ \pm}^{\prime}\left(0, \Lambda_{n}^{R}\right) \frac{d U_{ \pm}}{d \lambda}\left(0, \Lambda_{n}^{R}\right)=\left\|U_{ \pm}\left(\cdot, \Lambda_{n}^{R}\right)\right\|_{L_{2}\left(e_{ \pm}\right)}^{2}>0
$$


Assume first that $U_{ \pm}\left(0, \Lambda_{n}^{R}\right)=0$. Then it follows from 6.4 that $U_{ \pm}^{\prime}\left(0, \Lambda_{n}^{R}\right) \neq 0$. It is clear that

$$
\frac{d F}{d \lambda}\left(\Lambda_{n}^{R}\right)=U_{+}^{\prime}\left(0, \Lambda_{n}^{R}\right) \frac{d U_{-}}{d \lambda}\left(0, \Lambda_{n}^{R}\right)+U_{-}^{\prime}\left(0, \Lambda_{n}^{R}\right) \frac{d U_{+}}{d \lambda}\left(0, \Lambda_{n}^{R}\right)
$$

The obtained identities imply formula 6.2 for the derivative of the function $F(\lambda)$. Moreover, the quotients $\frac{U_{+}^{\prime}\left(0, \Lambda_{n}^{R}\right)}{U_{-}^{\prime}\left(0, \Lambda_{n}^{R}\right)}$ and $\frac{U_{-}^{\prime}\left(0, \Lambda_{n}^{R}\right)}{U_{+}^{\prime}\left(0, \Lambda_{n}^{R}\right)}$ are of the same sign and this is why the derivative of the function $F$ at the point $\Lambda_{n}^{R}$ is non-zero.

We proceed to the case $U_{ \pm}\left(0, \Lambda_{n}^{R}\right) \neq 0$. Then it follows from equation 2.18 that

$$
U_{ \pm}^{\prime}\left(0, \Lambda_{n}^{R}\right)=(\alpha+\beta) U_{ \pm}\left(0, \Lambda_{n}^{R}\right)-U_{\mp}^{\prime}\left(0, \Lambda_{n}^{R}\right) \frac{U_{ \pm}\left(0, \Lambda_{n}^{R}\right)}{U_{\mp}\left(0, \Lambda_{n}^{R}\right)} .
$$

Employing these identities, by straightforward calculations we check

$$
\begin{aligned}
& \frac{d\left(F-(\alpha+\beta) U_{-}(0, \cdot) U_{+}(0, \cdot)\right)}{d \lambda}\left(\Lambda_{n}^{R}\right)=U_{+}^{\prime}\left(0, \Lambda_{n}^{R}\right) \frac{d U_{-}}{d \lambda}\left(0, \Lambda_{n}^{R}\right)+U_{-}^{\prime}\left(0, \Lambda_{n}^{R}\right) \frac{d U_{+}}{d \lambda}\left(0, \Lambda_{n}^{R}\right) \\
&+ U_{-}\left(0, \Lambda_{n}^{R}\right) \frac{d U_{+}^{\prime}}{d \lambda}\left(0, \Lambda_{n}^{R}\right)+U_{+}\left(0, \Lambda_{n}^{R}\right) \frac{d U_{-}^{\prime}}{d \lambda}\left(0, \Lambda_{n}^{R}\right) \\
&-(\alpha+\beta) U_{-}\left(0, \Lambda_{n}^{R}\right) \frac{d U_{+}}{d \lambda}\left(0, \Lambda_{n}^{R}\right)-(\alpha+\beta) U_{+}\left(0, \Lambda_{n}^{R}\right) \frac{d U_{-}}{d \lambda}\left(0, \Lambda_{n}^{R}\right) \\
&=-\frac{U_{+}\left(0, \Lambda_{n}^{R}\right)}{U_{-}\left(0, \Lambda_{n}^{R}\right)} U_{-}^{\prime}\left(0, \Lambda_{n}^{R}\right) \frac{d U_{-}}{d \lambda}\left(0, \Lambda_{n}^{R}\right)+U_{+}\left(0, \Lambda_{n}^{R}\right) \frac{d U_{-}^{\prime}}{d \lambda}\left(0, \Lambda_{n}^{R}\right) \\
&-\frac{U_{-}\left(0, \Lambda_{n}^{R}\right)}{U_{+}\left(0, \Lambda_{n}^{R}\right)} U_{+}^{\prime}\left(0, \Lambda_{n}^{R}\right) \frac{d U_{+}}{d \lambda}\left(0, \Lambda_{n}^{R}\right)+U_{-}\left(0, \Lambda_{n}^{R}\right) \frac{d U_{+}^{\prime}}{d \lambda}\left(0, \Lambda_{n}^{R}\right) \\
&= \frac{U_{+}\left(0, \Lambda_{n}^{R}\right)}{U_{-}\left(0, \Lambda_{n}^{R}\right)}\left(U_{-}\left(0, \Lambda_{n}^{R}\right) \frac{d U_{-}^{\prime}}{d \lambda}\left(0, \Lambda_{n}^{R}\right)-U_{-}^{\prime}\left(0, \Lambda_{n}^{R}\right) \frac{d U_{-}}{d \lambda}\left(0, \Lambda_{n}^{R}\right)\right) \\
&+\frac{U_{-}\left(0, \Lambda_{n}^{R}\right)}{U_{+}\left(0, \Lambda_{n}^{R}\right)}\left(U_{+}\left(0, \Lambda_{n}^{R}\right) \frac{d U_{+}^{\prime}}{d \lambda}\left(0, \Lambda_{n}^{R}\right)-U_{+}^{\prime}\left(0, \Lambda_{n}^{R}\right) \frac{d U_{+}}{d \lambda}\left(0, \Lambda_{n}^{R}\right)\right) .
\end{aligned}
$$

By formulae 6.4 this implies identity 6.3). Here the quotients $\frac{U_{+}\left(0, \Lambda_{n}^{R}\right)}{U_{-}\left(0, \Lambda_{n}^{R}\right)}$ and $\frac{U_{-}\left(0, \Lambda_{n}^{R}\right)}{U_{+}\left(0, \Lambda_{n}^{R}\right)}$ are of the same sign and this is why the right hand side of identities (6.3) does not vanish. The proof is complete.

Let us find the spectrum of the operator $\mathcal{H}_{\varepsilon}^{R}$. We seek the eigenfunctions of the operator $\mathcal{H}_{\varepsilon}^{R}$ as

$$
\Psi_{\varepsilon}^{R}(x):= \begin{cases}C_{-} U_{-}\left(x_{-}, \lambda\right) & \text { on } e_{-}, \\ C_{+} U_{+}\left(x_{+}, \lambda\right) & \text { on } e_{+}, \\ C_{\varepsilon} U_{R}\left(\frac{x_{\varepsilon}}{\varepsilon}, \varepsilon, \varepsilon^{2} \lambda\right) & \text { on } e_{\varepsilon},\end{cases}
$$

where $C_{ \pm}, C_{\varepsilon}$ are some constants. Again, it is sufficient to check the condition at the point $M_{0}$ for such functions; this leads us to the system of linear equations:

$$
\left\{\begin{array}{l}
C_{-} U_{-}(0, \lambda)-C_{+} U_{+}(0, \lambda)=0, \\
C_{-} U_{-}(0, \lambda)-C_{\varepsilon} U_{R}\left(0, \varepsilon, \varepsilon^{2} \mu\right)=0, \\
C_{-}\left(U_{-}^{\prime}(0, \lambda)+\alpha U_{-}(0, \lambda)\right)+C_{+} U_{+}^{\prime}(0, \lambda)+C_{\varepsilon} \varepsilon^{-1} U_{R}^{\prime}\left(0, \varepsilon, \varepsilon^{2} \lambda\right)=0 .
\end{array}\right.
$$

Equating the determinant of this system to zero, we arrive to the equation for the eigenvalues of the operator $\mathcal{H}_{\varepsilon}^{R}$ :

$$
G^{R}(\lambda, \varepsilon)=0
$$


where

$$
\begin{aligned}
G^{R}(\lambda, \varepsilon) & :=U_{R}\left(0, \varepsilon, \varepsilon^{2} \lambda\right)\left(F(\lambda)-\alpha U_{-}(0, \lambda) U_{+}(0, \lambda)\right)+\varepsilon^{-1} U_{R}^{\prime}\left(0, \varepsilon, \varepsilon^{2} \lambda\right) U_{-}(0, \lambda) U_{+}(0, \lambda) \\
& =\left(F(\lambda)-(\alpha+\beta) U_{-}(0, \lambda) U_{+}(0, \lambda)\right)+A(\lambda, \varepsilon) U_{-}(0, \lambda) U_{+}(0, \lambda), \\
A(\lambda, \varepsilon) & :=\varepsilon^{-1} U_{R}^{\prime}\left(0, \varepsilon, \varepsilon^{2} \lambda\right)+\beta U_{R}\left(0, \varepsilon, \varepsilon^{2} \lambda\right) .
\end{aligned}
$$

By relations (4.1), (4.2), the function $A$ is holomorphic in $\lambda$ and $\varepsilon$ and the identities hold:

$$
A\left(\Lambda_{n}^{R}, 0\right)=0, \quad \frac{d A}{d \varepsilon}\left(\Lambda_{n}^{R}, 0\right)=\beta \phi_{1}(0)+\phi_{2}^{\prime}(0)+\Lambda_{n}^{R} \phi_{3}^{\prime}(0) .
$$

This is why the function $G^{R}$ is holomorphic in $\lambda$ and $\varepsilon$. It is clear that equation (6.5) holds as $\Lambda=\Lambda_{n}^{R}, \varepsilon=0$. This implies immediately that the roots of equation 6.5 converge to $\Lambda_{n}^{R}$ as $\varepsilon \rightarrow+0$.

Let us find out the structure of the function $G^{R}$ in the vicinity of the points $\lambda=\Lambda_{n}^{R}$. First we consider the case $U_{ \pm}\left(\Lambda_{n}^{R}\right)=0$. Here by 6.2 and relations 4.1 we have

$$
\frac{d G^{R}}{d \lambda}\left(\Lambda_{n}^{R}, 0\right)=\frac{d F}{d \lambda}\left(\Lambda_{n}^{R}\right) \neq 0
$$

and by the implicit function theorem, equation $(6.5)$ possesses the unique solution. By straightforward substitution we infer easily that $\lambda=\Lambda_{n}^{R}$ is a solution of equation (6.5) for all considered values $\varepsilon$. This proves the theorem in the case $U_{ \pm}\left(\Lambda_{n}^{R}\right)=0$.

Assume now that $U_{ \pm}\left(\Lambda_{n}^{R}\right) \neq 0$. In this case by 6.3$\left.), 6.6\right)$ we have

$$
\frac{d G^{R}}{d \lambda}\left(\Lambda_{n}^{R}, 0\right)=\frac{d\left(F-(\alpha+\beta) U_{-}(0, \cdot) U_{+}(0, \cdot)\right)}{d \lambda}\left(\Lambda_{n}^{R}\right) \neq 0
$$

and by the implicit function theorem there exists the unique solution $\lambda_{n}^{R}(\varepsilon)$ of equation 6.5 ) holomorphic in $\varepsilon$ and converging to $\Lambda_{n}^{R}$ as $\varepsilon \rightarrow+0$. The derivative of this solution is expressed by the formula similar to 5.5$)$ :

$$
\frac{d \lambda_{n}^{R}}{d \varepsilon}(0)=-\frac{\frac{d G^{R}}{d \varepsilon}\left(\Lambda_{n}^{R}, 0\right)}{\frac{d G^{R}}{d \lambda}\left(\Lambda_{n}^{R}, 0\right)}=-\frac{\frac{d A}{d \varepsilon}\left(\Lambda_{n}^{R}, 0\right)}{\frac{d\left(F-(\alpha+\beta) U_{-}(0, \cdot) U_{+}(0, \cdot)\right)}{d \lambda}\left(\Lambda_{n}^{R}\right)},
$$

where we have also employed formula (6.7). Let us calculate the denominator in this formula. We have

$$
\begin{gathered}
\phi_{2}^{\prime}(0)=-\int_{0}^{1} W_{0}(t) d t-\int_{0}^{1} W_{-1}(t) \phi_{1}(t) d t \\
-\int_{0}^{1} W_{-1}(t) \phi_{1}(t) d t=-\int_{0}^{1} \phi_{1}^{\prime \prime}(t) \phi_{1}(t) d t=\phi_{1}(0) \phi_{1}^{\prime}(0)+\int_{0}^{1}\left(\phi_{1}^{\prime}(t)\right)^{2} d t \\
=-\beta \phi_{1}(0)+\int_{0}^{1}\left(\int_{t}^{1} W_{-1}(s) d s\right)^{2} d t
\end{gathered}
$$

Substituting the obtained identities and (6.3), (6.6) into (6.8), we arrive at formula (2.19). The proof of Theorem 2.4 is complete.

\section{ACKNOWLEDGEMENTS}

This work was initiated during a visit of D.I. Borisov to Al-Farabi Kazakh National University, Almaty, Kazakhstan in December, 2018. He is deeply grateful for warm hospitality extended to him during the visit. 


\section{BIBLIOGRAPHY}

1. D. Borisov. On a $\mathcal{P} \mathcal{T}$-symmetric waveguide with a pair of small holes // Trudy Inst. Matem. Mekh. UrO RAN. 18:2, 22-37 (2012). [Proc. Steklov Inst. Math. 281. Suppl 1. 5-21 (2013).]

2. D.I. Borisov, A.I. Mukhametrakhimova. On norm resolvent convergence for elliptic operators in multi-dimensional domains with small holes // Probl. Matem. Anal. 92, 69-81 (2018). [J. Math. Sci. 232:3, 283-298 (2018).]

3. A.M. Il'in. Matching of asymptotic expansions of solutions of boundary value problems. Nauka, Moscow (1989). [Am. Math. Soc., Providence, RI (1992).]

4. V.G. Maz'ya, S. A. Nazarov, B. A. Plamenevskii. Asymptotic expansions of the eigenvalues of boundary value problems for the Laplace operator in domains with small holes // Izv. AN SSSR. Ser. Matem. 48:2, 347-371 (1984). [Math. USSR-Izv. 24:2 (1985), 321-345.]

5. Yu.V. Pokornyǐ, O.M. Penkin, V.L. Pryadiev, A.V. Borovskikh, K.P. Lazarev, S.A. Shabrov. Differential equations on geometric graphs. Fizmatlit, Moscow (2005).

6. S. Albeverio, F. Gesztesy, R. Høegh-Krohn, H. Holden. Solvable models in quantum mechanics. AMS Chelsea Publ., Amer. MAth. Soc., Providence, Rhode Island (2000).

7. G. Berkolaiko, P. Kuchment. Introduction to quantum graphs, Amer. Math. Soc., Providence, RI (2013).

8. G. Berkolaiko, Y. Latushkin, S. Sukhtaev. Limits of quantum graph operators with shrinking edges // Preprint: arXiv:1806.00561 (2018)

9. T. Cheon, P. Exner, O. Turek. Approximation of a general singular vertex coupling in quantum graphs // Ann. Phys. 325:3, 548-578 (2010).

Denis Ivanovich Borisov,

Institute of Mathematics,

Ufa Federal Research Center, RAS,

Chernyshevky str. 112,

450008, Ufa, Russia

Bashkir State University,

Zaki Validi str., 3a,

450000, Ufa, Russia

University of Hradec Králové,

Rokitanskeho, 62

50003, Hradec Králové, Czech Republic

E-mail: BorisovDI@yandex.ru

Maral Nurlanovna Konyrkulzhaeva,

Al-Farabi Kazakh National University,

Al-Farabi av. 71,

A15E3B4, Almaty, Kazakhstan

E-mail: maralkulzha@gmail.com 